\title{
Research on the Demand of Visual Identification System Design for Railway Passenger Stations
}

\author{
Duan Wenjie \\ Changchun University of Science and Technology, Changchun, 130022, China
}

Keywords: Visual identification system, Railway passenger station, Application

\begin{abstract}
With the rapid development of railway construction, a large number of newly-built railway passenger stations have been gradually put into operation. In order to establish a visual recognition system more suitable for the high-speed rail passenger station, this paper demonstrates the visual recognition system of the railway passenger station by exploring and researching the existing passenger stations in China. This paper mainly analyzes the significance of visual recognition system in the context of shortening passengers' dwell time, walking path and improving the service quality of railway system.
\end{abstract}

\section{Introduction}

With the continuous development of the social economy, China's transportation industry is in the same stage of rapid development as China's economy. At this stage, inter-city transportation such as aviation, railways, and highways is becoming more and more connected to public transportation such as public transportation, subways, and light rail. The construction of an integrated transportation hub system that integrates multiple modes of transportation has become the first choice for large cities to develop modern transportation. At present, the construction of such a diversified large-scale comprehensive transportation hub has entered a period of rapid development. Under such

As an important node of passenger distribution, the design of the visual identity system of the high-speed railway station should be based on the prominent guiding function, to improve the humanized service, give full play to its humanistic care role, and also bear the responsibility of cultural communication and display of the city image. The high-speed rail station has become a transportation hub with a relatively single function, and has become a transportation hub integrating multiple modes of transportation. It has greatly facilitated passengers and increased the complexity of its functions. Passengers are unable to find the right path in a complex environment, which increases the length of stay and the length of the inquiry. Therefore, it is necessary to use a personalized and humanized service concept to establish a convenient visual identification system in modern high-speed rail passenger transportation, in order to guide the inbound and outbound passengers to the correct path and alleviate the pressure of passenger gathering in the passenger station.

\section{Overview of railway visual identification system}

Visual recognition is a visual display designed as text or graphics. It is a tool for conveying information in text and visual graphics. The visual identity system is a space guidance system that is set up to deliver information to people who are unfamiliar with the environment, easily lost or helpless. The goal is to enable people to get accurate information and the right path in the shortest amount of time in an increasingly complex space environment. Compared with language, visual recognition has a stronger visual impact, has a larger amount of information, and can convey information more quickly, accurately, and more strongly. As a kind of traffic building, the modern railway passenger station itself needs to move and evacuate the inbound and outbound people in a large amount of time in a short period of time, reducing the invalid stay time of the users. Therefore, the visual recognition system plays a very important role in the traffic function of the railway 
passenger station.

The visual identification system (VI) of the railway passenger station is the core of the UIS of the passenger station image recognition system. The UIS (UniversityIdentity System) consists of three organic components: 1MI (Mind Identity) concept recognition, such as the service concept, value orientation and service tenet of the railway passenger station; 2BI (Behaviour Identity) behavior recognition, such as railway The development of the passenger station enterprise development strategy, the direction of development, various behavioral norms of railway employees, etc.; 3VI (Visual Identity) visual identification, such as basic systems (flags, standard words, auxiliary graphics, etc.) and application systems (light boxes, environmental indicators) System, etc.). The concept recognition system, behavior recognition system and enterprise identification system of the railway passenger station have different directional and essential characteristics, so the symbols expressed by the visuals are not likely to completely follow the design of the enterprise. Railway passenger operation is China's iconic state-owned enterprise. Apart from being affected by the market economy, it is a product of economic and cultural conditions. Therefore, the visual identification system of the railway passenger station also has its own unique characteristics.

\section{Design functions and principles of visual recognition system for railway passenger stations}

\subsection{Functional principles of the visual identity system of the high-speed railway station}

The design of the visual identity system of the high-speed railway station not only has the functions of guidance, instructions and explanations of the environment, but also has the functions of attracting attention, warnings and mandatory commands. At the same time, it is also an important part of the high-speed rail station environment, an important part of creating a high-speed rail station style and shaping the culture of the high-speed rail station. The function of the visual identity system of the high-speed rail station is mainly reflected in the transmission of text, graphics and color, conveying information to passengers with concise and clear visual language symbols, and ensuring that the information is easy to identify, has no communication barriers, and has clear meaning and unambiguity. It should lead passengers effectively complete a series of related actions in accordance with the guiding instructions in the high-speed rail station.

\subsection{Graphic recognition function of visual recognition system of high-speed railway station}

Graphics can be described as the soul of the visual recognition system. It is a cross-border, cross-cultural international language that relies on human intuition to communicate information. Graphic communication not only has a much larger information carrying capacity than text transmission, but also has an advantage in the speed of transmission and is visually appealing. The graphic design in the visual identity system of the high-speed railway station should pay attention to the relationship between representation and abstraction, universality and particularity, cosmopolitan and regional.

\subsection{Text recognition function of visual recognition system of high-speed railway station}

The text in visual recognition system is irreplaceable in the completeness and accuracy of information. On the one hand, the content of the text should be concise and clear, and the expression should be clear and standard, so that the audience can easily understand the text content. On the other hand, using the Chinese standard and the Pinyin specification, and the English, the text information recognition function can be maximized. On the font of the text, use the no-line font to improve recognition validity. The no-line font was officially used by the British government in the subway identification system in 1316. Since the no-line font is concise and clear, the communication effect is good. So far, the no-line font has been adopted by most public transportation identification systems. On the scale of the text, it is necessary to conform to the laws of human visual physiology. There is a uniform standard ratio between the scale of the text and the distance between the human and the text. 


\subsection{Reasonable design of the visual identification system of the high-speed railway station}

The visual identification system of the high-speed railway station must be scientific, first of all to reflect the rationality in the design. The design is for people. The so-called rationality of design is a word for a pair of passengers. Therefore, the rationality of the visual identity system of the high-speed railway station should conform to the physiological characteristics, psychological characteristics and behavioral habits of people. Location setting of the visual identity system of the high-speed rail station. The guide sign is an important carrier for the visual identity system of the high-speed rail station. The rationality of the position setting of the guiding sign is closely related to the visual physiology and visual habits of the person, and its reasonableness directly affects the guiding effect of the guiding card. Therefore, it is necessary to set a suitable sign at a suitable point. The personality of the visual identity system of the high-speed rail station. To effectively realize the function of the high-speed rail visual identity system, it is necessary to ensure that it can be used normally. This not only requires the material and process of the visual recognition system carrier to have good anti-destructive properties, but also ensures that its structure, dimensions, materials and the like have individuality. At the same time, the guiding facilities with safety hazards should be adjusted and repaired in time to avoid unnecessary damage.

\section{The demand for visual recognition system design in railway passenger station}

Due to the functional characteristics of the modern railway passenger station, the visual recognition system also has special requirements for the environmental indication system.

\subsection{Changes in design concepts for static identification systems}

The environmental indicator system in the building serves as a space guidance facility within the building and is closely related to the design of the building space. Therefore, the changes in the architectural design concept of the modern railway passenger station will also have a profound impact on the design of the passenger station environmental indicators. The modern railway passenger station is gradually changing from a single passenger function in the past to an important node of an integrated transportation hub, and more and more integrated with the traffic planning of the entire city. Today's large-scale railway passenger station is not only a window of the city, but also a link between the city's external transportation links and the transfer nodes of various modes of transportation within the city. This requires that the environmental indication system of the railway passenger station must provide passengers with convenient travel guidance to minimize passenger inquiry times and meet passenger travel requirements. Establish a scientific, standardized, people-oriented environmental indicator system that allows passengers to accurately understand relevant travel information without asking questions.

\subsection{The special operational characteristics of the railway passenger station The demand for the environmental indication system}

The operational characteristics of the modern railway passenger station are concentrated in convenience and efficiency. Therefore, it is also required that the railway passenger station should strengthen the "passivity" as much as possible. The travel time of ordinary passengers is not only the time of taking the train, but also the time of purchase of passengers at the railway station, the time of finding the road, the waiting time, the transfer time and the time of the city. The purpose of establishing an integrated transportation hub around the modern railway passenger station is to compress the ineffective time spent by passengers in the above process as much as possible, improve passenger travel efficiency, avoid the intersection of passengers entering and leaving the station, and reduce the load on the railway passenger station. This also requires that the railway passenger station must establish an effective environmental indication system, organically link the different spaces of the station through the identification and guidance system, regulate the passenger's behavior path, reduce the passenger's pause time, and enable the passengers in the station to have a good order of activity to avoid security problems caused by poor order. 


\subsection{Improvement of Service Quality of Railway Passenger Stations Demand for Environmental Indicator System}

Due to the country's strong support for railway development, the railway system hardware facilities have been significantly improved in recent years, effectively increasing the capacity and improving the passenger environment. The quality of railway services has also undergone a fundamental change. The services provided by the modern railway passenger station to passengers include the whole process from the start of ticket purchase to the departure of the bus, the arrival of the bus to the station and the completion of the transfer. This requires modern railway passenger stations to reduce the invalid time of passengers in the station from the optimization of passenger behavior process. It is necessary to establish a reasonable environmental indication system to enable passengers to independently and independently complete the process of purchasing tickets, entering stations, waiting for cars, checking tickets, passing down, and leaving stations, thereby reducing passengers' time and physical exertion. This is a reflection of the respect for passenger autonomy by the modern railway passenger station and the improvement of the quality of railway passenger transport services.

\section{Constructing a modern high-speed rail passenger-oriented visual recognition system}

The static identification system of the railway passenger station is a static information that provides space guidance in the specific space environment of railway transportation. It guides passengers in and out of the passenger station in a textual and graphical and intuitive form to realize the transfer.

\subsection{Taking the basic behavior of passengers as the guiding path}

The design of the static identification system should be based on the convenience of the passenger's behavior, and its layout must be based on the passenger's basic behavioral path. Taking the passenger station streamline as an example, the entrance signage guidance in the railway passenger station should follow the basic route of the station-sales office-inbound hall-waiting hall-station platform to reduce the walking distance and shorten the passenger's walking distance in the station. The length of time the passenger stays in the station.

From the perspective of the passengers in the internal space environment of the station, it is important to set the guides at the connection points and forks of the various space environments. Most passengers will be confused in these two positions. I don't know where to go. The signs set here need to clearly and clearly indicate the direction of space and avoid passengers' stay. For example, the connection point between the ticket office and the waiting room or the transition space should clearly indicate the direction of the two spaces, and indicate the direction of the waiting room at the exit of the ticket office. Instead, mark the direction of the ticket office at the exit of the waiting room to avoid the phenomenon that the passengers look for the next space direction sign after leaving a space.

From the passenger outbound point of view, to allow passengers to quickly get the exit sign in the outbound direction after the high-speed rail train, it is necessary to place a clear outbound sign on the platform level. At present, the situation of most stations is that passengers only go with the flow after getting off the bus. The direction of the specific flow is correct or not. It is not clear where most passengers are concerned. It is inevitable that there will be uneasy psychological conditions. After the passenger leaves the station, the passenger should immediately obtain the information of the transfer mode and direction, and place the direction guide sign of the transfer vehicle and the surrounding environment direction guide sign in the outbound or outbound passage to enhance the direction of the passenger in the strange space. Psychology relieves their anxiety and fear.

\subsection{Design principles that are easy to understand}

Most of the passengers who choose to take the train are unfamiliar with the railway passenger 
station. They may carry more luggage, have inconvenient mobility, and may have time to catch the train. These all put forward high requirements for the systemicity, integrity and identifiability of the identification information.

The signage guidance system must be placed in the most prominent position during the passenger's travel, providing the passenger with the information most needed for the location, allowing the passenger to identify the layout information of the logo in a relatively complex environment. The logo should be set at an appropriate height and angle to avoid being blocked by other objects, and not too high to affect the identification.

The layout information of the logo should be uniformly designed in terms of graphic symbols and font width and stroke thickness. The functional requirements of the logo in the railway passenger station are far greater than the artistic requirements. Therefore, the logo font should not be handwritten and highly modified. It must conform to the passenger's cognitive habits and reflect the unique rigor of the railway passenger station as a traffic building.

The object of the static identification system service is all passengers, and the age, culture and cognitive ability of the passengers are different. At the same time, as a core component of the city's comprehensive transportation hub, the modern railway passenger station is more likely to come from different countries and regions. This requires that the identification system should adopt standardized and easily recognized graphic symbols and apply Chinese and English bilingual forms. . The logo should also take into account the travel-oriented needs of socially disadvantaged groups such as the old, the weak, the sick and the disabled.

\subsection{Guarantee the continuity of space guidance}

The signage guidance system should be a complete system. From the first sight of the passengers entering the station, the passengers should be provided with self-service guidance services until the passengers board or change. It should be ensured that all nodes that may cause route ambiguity have clear and clear marking guidance, so that the marking guidance system forms a coherent system to avoid the formation of blind spots to ensure the continuity of passengers' walking. Therefore, the setting of the identification system point can not only consider the isolated setting of one identification, but should carry out unified planning of the front and rear identification, and integrate the design to form the space guidance.

\section{Conclusion}

In summary, with the constant changes of the times, the rapid development of economy, culture and science and technology, the railway industry is also advancing constantly, and the visual recognition system of the railway passenger station will become a more mature and more perfect design system. The form is more beautiful, the content is more abundant, the design principle is more profound, and the characteristics of the environmental indicator system of the railway passenger station will be more distinct.

\section{References}

[1] Zhang $\mathrm{J} \mathrm{M}, \mathrm{Xu} \mathrm{J}, \mathrm{Xu} \mathrm{G}$, et al. Research on the Design Method of the Visual Image of Energy-Saving and Environmental Protection Product[J]. Applied Mechanics \& Materials, 2014, 543-547:4121-4124.

[2] Yu F. Research on Static Sign-oriented Identification System Design for Passenger Stations__Based on Design of Sign-oriented System of Pingyao East Station[J]. Railway Standard Design, 2011.

[3] Yang Z. Research on Rapid Design System for Bend Door of Railway Vehicles[J]. Value Engineering, 2010.

[4] Zhou J. Research on the Design of Auxiliary Graphics in Visual Identity and Its Scalability[C]// International Conference on Smart Grid and Electrical Automation. IEEE Computer Society, 
2017:419-422.

[5] Abdullah, M. A. (2011). System identification of railway trains pantograph for active pantograph simulation. Journal of System Design \& Dynamics,5(5), 1141-1154.

[6] Belhumeur, P. N., Chen, D., Feiner, S., Jacobs, D. W., Kress, W. J., \& Ling, H., et al. (2008). Searching the World's Herbaria: A System for Visual Identification of Plant Species. Computer Vision - ECCV 2008, European Conference on Computer Vision, Marseille, France, October 12-18, 2008, Proceedings (Vol.5305, pp.116-129). DBLP. 